\title{
Graphene-assisted matrix solid-phase dispersion for extraction of polybrominated diphenyl ethers and their methoxylated and hydroxylated analogs from environmental samples
}

\author{
Qian Liu, Jianbo Shi, Jianteng Sun, Thanh Wang, Lixi Zeng, Nali Zhu, Guibin Jiang* \\ State Key Laboratory of Environmental Chemistry and Ecotoxicology, Research Center for Eco-Environmental Sciences, Chinese Academy of Sciences, Beijing 100085, China
}

\section{A R T I C L E I N F O}

\section{Article history:}

Received 5 July 2011

Received in revised form

13 September 2011

Accepted 13 September 2011

Available online 19 September 2011

\section{Keywords:}

Graphene

Matrix solid-phase dispersion

Extraction

Polybrominated diphenyl ether

Methoxylated polybrominated diphenyl

ether

Hydroxylated polybrominated diphenyl ether

\begin{abstract}
A B S T R A C T
In this work, we developed a novel graphene-assisted matrix solid-phase dispersion (GA-MSPD) method for extraction of polybrominated diphenyl ethers (PBDEs) and their methoxylated (MeO-) and hydroxylated ( $\mathrm{OH}-)$ analogs from environmental samples. We found that grinding the solid sample with chemically converted graphene (CCG) powder yielded a tight contact and sufficient dispersion of the sample matrix due to the large surface area and flexible nanosheet morphology of CCG. The resultant blend was eluted using a two-step elution strategy: PBDEs and MeO-PBDEs were eluted firstly by hexane/dichloromethane and analyzed by GC-ECD, and then OH-PBDEs were eluted by acetone and determined by LC-ESI-MS/MS. The GA-MSPD conditions were optimized in detail. Better recoveries were obtained with GA-MSPD than with other sorbents (C18 silica, Florisil and carbon nanotubes) and other extraction techniques (Soxhlet and accelerated solvent extraction). Other advantages of GA-MSPD, including reduced consumption of sorbent and solvent, good selectivity and short extraction time, were also demonstrated. In analysis of soil samples, the method detection limits of five PBDEs, ten MeO-PBDEs and ten OH-PBDEs were in the range of 5.9-28.7, 14.3-46.6, and 5.3-212.6 $\mathrm{pg} \mathrm{g}^{-1}$ dry weight, respectively. The proposed method was successfully applied to the extraction of PBDEs, MeO-PBDEs and OH-PBDEs from different kinds of spiked environmental samples, including soil, tree bark and fish.
\end{abstract}

(c) 2011 Elsevier B.V. All rights reserved.

\section{Introduction}

Graphene, a two-dimensional nanomaterial consisting of a single-layer of $\mathrm{sp}^{2}$ network of carbon atoms, has stimulated great interest in the scientific community due to its exceptional electronic, mechanical and thermal properties [1-3]. A particularly attractive feature of graphene is the ultrahigh surface area (theoretical value $2630 \mathrm{~m}^{2} \mathrm{~g}^{-1}$ [4]), which makes graphene a promising sorbent material with high sorption capacity. Its double-sided polyaromatic scaffold with large delocalized $\pi$-electron system also favors its use as sorbent material. Furthermore, bulk quantities of graphene as either colloid dispersions or powders can be readily produced from graphite by chemical approaches at relatively low costs [5,6], and the resultant graphene is usually designated as chemically converted graphene (CCG). This renders the possibility of using relatively large amounts of graphene in adsorbent applications. For instance, CCG-based materials have been used for removal of contaminants from drinking waters [7,8]. We have recently demonstrated the use of CCG as SPE adsorbent in classic

\footnotetext{
* Corresponding author. Fax: +86106284 9937.

E-mail address: gbjiang@rcees.ac.cn (G. Jiang).
}

cartridge format for extraction of chlorophenols from environmental waters [9]. Graphene and graphene oxide (GO) supported on silica have also been shown to be excellent adsorbents for reversedphase and normal-phase SPE, respectively [10]. Dong et al. [11] and Tang et al. [12] have reported the use of CCG or GO dispersed in sample solutions to preconcentrate small molecules or biomolecules. Applications of CCG in solid-phase microextraction (SPME) and stirbar sorptive extraction have also been reported [13-15]. However, until now, applications of graphene in sorption-related fields have still been relatively few, and its full potential has yet to be realized.

Polybrominated diphenyl ethers (PBDEs) are a class of brominated flame retardants (BFRs) used in a wide array of consumer products. They have received considerable attention over the past two decades because of their health risks [16] and ubiquity in wildlife and humans around the globe [17]. Recently, hydroxylated (OH-) and methoxylated (MeO-) PBDEs have also attracted much focus because their concentrations in marine biota can be higher than those of PBDEs [18]. These compounds have also been identified in human milk and blood $[19,20]$. Due to the associated health risks such as thyroid disruption and cytotoxicity of these PBDE analogs $[21,22]$, it is important to understand the sources and biotransformation processes of these compounds in the environment. However, this is not a straightforward task partly because there is 
still a lack of reliable and efficient methods for simultaneous analysis of PBDEs and their $\mathrm{MeO}-$ and $\mathrm{OH}-$ analogs in environmental and biological matrices. Particularly, the large differences in polarity and $K_{\text {ow }}$ make these compounds difficult to be extracted together efficiently.

In this work, we aimed to develop a rapid, simple and efficient graphene-assisted matrix solid-phase dispersion (GA-MSPD) method to extract PBDEs and their $\mathrm{MeO}-$ and $\mathrm{OH}-$ analogs from environmental matrices. This also represents the first attempt to use graphene as sorbent in MSPD. MSPD allows simultaneous sample dispersion, extraction and cleanup in a single step, thus eliminating most of the complications of classical liquid-liquid extraction or solid-phase extraction (SPE) when handling with solid samples $[23,24]$. The solid sample was mixed and ground with CCG powder to produce an apparent homogeneous blend. PBDEs and $\mathrm{MeO}$-PBDEs were eluted with hexane/dichloromethane (DCM) and subjected to gas chromatography-electron capture detection (GC-ECD) analysis, and then OH-PBDEs were eluted with acetone and analyzed by liquid chromatography-electrospray-tandem mass spectrometry (LC-ESI-MS/MS). The GA-MSPD conditions were optimized in detail, and the performance of the method was compared to other sorbents and other extraction techniques. Finally, the proposed method was validated with different real environmental matrices, including soil, tree bark and fish.

\section{Experimental}

\subsection{Chemicals and reagents}

Individual PBDE (BDE-17, 28, 47, 66, and 85), MeO-PBDE (3'-MeO-BDE-28, 4-MeO-BDE-42, 3-MeO-BDE-47, 5-MeO-BDE-47, 6-MeO-BDE-47, 4'-MeO-BDE-49, 2'-MeO-BDE-68, 6-MeO-BDE-85, $5^{\prime}-\mathrm{MeO}-\mathrm{BDE}-99$, and 6'-MeO-BDE-99) and OH-PBDE (3'-OH-BDE28, 4-OH-BDE-42, 3-OH-BDE-47, 5-OH-BDE-47, 6-OH-BDE-47, 4'-OH-BDE-49, 2'-OH-BDE-68, 6-OH-BDE-85, 5'-OH-BDE-99, and 6'-OH-BDE-99) standards were purchased from AccuStandard (New Haven, CT). Stock solutions of PBDEs and MeO-PBDEs were prepared in hexane at $1 \mu \mathrm{g} \mathrm{mL}^{-1}$, and those of OH-PBDEs were prepared in ACN at $1 \mu \mathrm{g} \mathrm{mL}^{-1}$. The stock solutions were stored in the dark at $4{ }^{\circ} \mathrm{C}$. CCG powder was synthesized by using the modified Hummers method followed by hydrazine reduction, as described in previous reports $[5,25]$. Florisil and C18 silica were purchased from Supelco (Bellefonte, PA). Before use, the Florisil was activated in an oven at $600^{\circ} \mathrm{C}$ for $4 \mathrm{~h}$ followed by $140^{\circ} \mathrm{C}$ for $6 \mathrm{~h}$. Multi-walled carbon nanotubes (MWCNTs, >98\%) were obtained from Nanotech Port Co. (Shenzhen, China). Anhydrous sodium sulfate was from Jinke (Tianjin, China) and dried at $660^{\circ} \mathrm{C}$ overnight before use. Sulfuric acid ( $\geq 96 \%$ ) was from Beijing Chemical Works (Beijing, China). Organic solvents used in this work were all from J. T. Baker (Phillipsburg, NJ) and of HPLC or pesticide grade. Ultrapure water was produced by a Milli-Q pure water system (Millipore, Billerica, MA). All reagents were of analytical grade unless otherwise noted.

\subsection{GA-MSPD procedure}

The GA-MSPD procedure is depicted in Fig. 1. Briefly, the sample was freeze-dried, homogenized and sieved through a 60 -mesh sieve to obtain the $<300 \mu \mathrm{m}$ fraction. Then, $100 \mathrm{mg}$ of the fraction was mixed with CCG powder $(10 \mathrm{mg})$ and ground in an agate mortar with an agate pestle for $5 \mathrm{~min}$ to yield an apparent homogeneous blend. The blend was transferred to a $1 \mathrm{~mL}$ SPE cartridge (Agilent, Santa Clara, CA) pre-packed with $50 \mathrm{mg}$ of anhydrous sodium sulfate and $50 \mathrm{mg}$ of Florisil as co-sorbent (see Fig. S1 in Supporting Information). The addition of co-sorbent was to retain polar interferences and partly remove lipid from the non-polar extract. Afterwards, the cartridge was compressed with an upper frit. After grinding, the mortar and pestle were rinsed twice with $500 \mu \mathrm{L}$ of hexane/DCM 1:1, and the rinse was collected and used as the first-step eluent solvent. In this step, PBDEs and $\mathrm{MeO}-\mathrm{PBDEs}$ were eluted from the column under gravitational flow. The eluate was evaporated under gentle nitrogen flow and reconstituted in $50 \mu \mathrm{L}$ hexane for GC-ECD analysis. Then, the column was dried by air and eluted with $1 \mathrm{~mL}$ of acetone. The acetone eluate containing $\mathrm{OH}-\mathrm{PBDEs}$ was also evaporated under nitrogen flow and reconstituted in $50 \mu \mathrm{L}$ of ACN for LC-ESI-MS/MS analysis.

\subsection{Chromatographic and MS conditions}

PBDEs and MeO-PBDEs were determined by an Agilent $6890 \mathrm{~N}$ Network GC system with an electron capture detector and a 7683B series injector. GC separations were performed using a J\&W DB-5MS capillary column $(30 \mathrm{~m} \times 0.25 \mathrm{~mm}$ i.d., $0.25 \mu \mathrm{m}$ film thickness). Helium (99.999\%) was used as carrier gas at a flow rate of $0.5 \mathrm{~mL} \mathrm{~min}^{-1}$. Samples $(2 \mu \mathrm{L})$ were injected in splitless mode. The oven program was as follows: start at $80^{\circ} \mathrm{C}(1 \mathrm{~min})$, first ramp at $10^{\circ} \mathrm{C} \mathrm{min}^{-1}$ to $260^{\circ} \mathrm{C}$ (held for $2 \mathrm{~min}$ ), second ramp at $2{ }^{\circ} \mathrm{C} \mathrm{min}-1$ to $300^{\circ} \mathrm{C}$ (held for $3 \mathrm{~min}$ ). Injector and detector temperatures were set at 250 and $300^{\circ} \mathrm{C}$, respectively. The data acquisition and analysis were performed by MSD ChemStation software.

Quantitation of OH-PBDEs was carried out on an Agilent 1290 Infinity LC coupled to an Agilent 6460 Triple Quadrupole mass spectrometer equipped with an ESI source working in negative ion mode. LC separations were achieved on a Dionex Acclaim RSLC $120 \mathrm{C} 18$ column $(2.1 \mathrm{~mm} \times 100 \mathrm{~mm}, 2.2 \mu \mathrm{m})$ with an Agilent Zorbax Eclipse XDB-C8 guard column $(2.1 \mathrm{~mm} \times 12.5 \mathrm{~mm}, 5 \mu \mathrm{m})$ at $40{ }^{\circ} \mathrm{C}$. The gradient elution program was as follows: starting at 55\% $\mathrm{ACN} / 45 \%$ water, increasing ACN to $75 \%$ in $20 \mathrm{~min}$, then decreasing ACN to $55 \%$ in $1 \mathrm{~min}$, and keeping constant for $5 \mathrm{~min}$. The flow rate was $0.38 \mathrm{~mL} \mathrm{~min}^{-1}$. The sample injection volume was $10 \mu \mathrm{L}$. MS detection was performed in multiple reaction monitoring (MRM) mode using the $[\mathrm{M}-\mathrm{H}]^{-}$ion as precursor. The Agilent Jet Stream technology was applied for thermal gradient focusing, which uses hot nitrogen to improve ion generation and desolvation. The optimized parameters of MRM for quantitation of OH-PBDEs are listed in Table S1. Nitrogen was used as desolvation, nebulizing and collision gas. The sheath gas temperature was $400^{\circ} \mathrm{C}$ and the flow rate was $11 \mathrm{~L} \mathrm{~min}^{-1}$. The desolvation and nebulizing gas temperature was $350{ }^{\circ} \mathrm{C}$ and the flow rate was $5 \mathrm{~L} \mathrm{~min}^{-1}$ with a pressure of 50 psi. The capillary voltage was set at $5000 \mathrm{~V}$, and the nozzle voltage was $500 \mathrm{~V}$. The data acquisition and analysis were processed by MassHunter WorkStation software.

\subsection{Environmental samples}

Agricultural soils, tree barks of Cinnamomum camphora and mackerel fishes were all collected from an electronic waste (Ewaste) dismantling area in Taizhou, Zhejiang Province, China. The samples were homogenized and completely freeze-dried. The dried samples were then homogenized again and stored at $-20^{\circ} \mathrm{C}$. In the optimization experiments, the blank soils were collected in Beijing, China and pre-analyzed to confirm the concentrations of all the target compounds below detection limits. The samples were spiked with standards as follows [26]: the standard solutions of known concentration of the target analytes were prepared in acetone. The standard solutions were spiked to the dried samples, and then the spiked samples were homogenized and kept covered in a refrigerator at $4{ }^{\circ} \mathrm{C}$ overnight to allow sufficient dispersion and equilibration. 


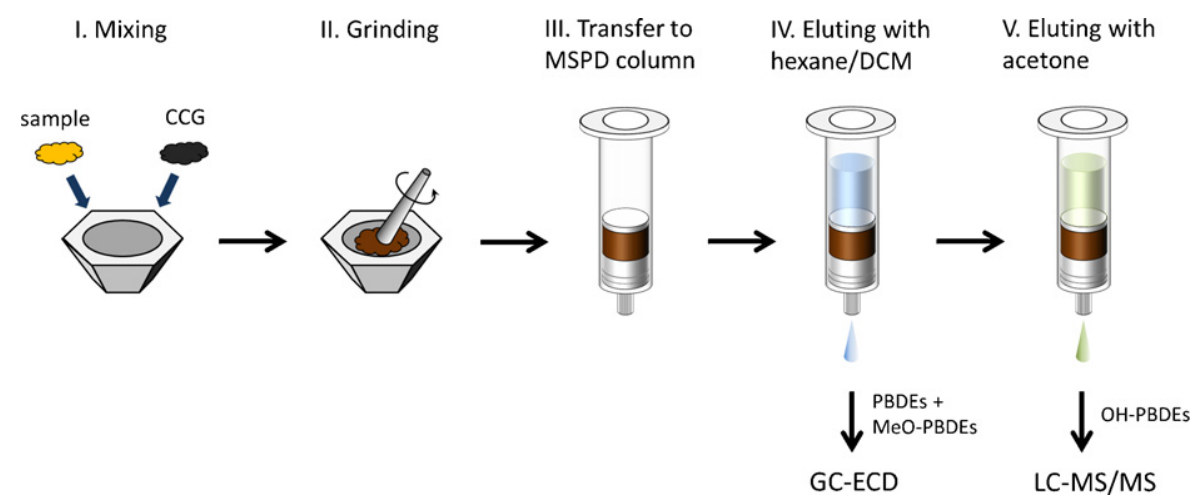

Fig. 1. Scheme showing the GA-MSPD procedure.

\section{Results and discussion}

\subsection{Characterization of CCG and CCG-soil blend}

In order to investigate the mechanism of GA-MSPD, we first characterized CCG and CCG-sample blend by SEM and TEM. Highresolution SEM image (Fig. 2A) showed CCG as a disordered solid consisting of semi-transparent and corrugated sheets. The thickness of the sheets judged from the edge was less than $2 \mathrm{~nm}$, indicating the predominance of single- or few layer of CCG sheets. This morphology of nanosheet was also confirmed by TEM (Fig. S2). Compared with other commonly used sorbents such as C18 silica and Florisil, CCG was relatively softer and more flexible. Fig. 2B showed the SEM image of ground soil sample, which consisted of discrete particles with irregular shapes at different sizes. After grinding with CCG, as shown in Fig. 2C, the soil particles were densely wrapped by randomly aggregated CCG sheets. Fig. 2D showed a semi-transparent CCG sheet attached on the surface of a soil particle. From Fig. 2E, the TEM image also showed that the ground soil particles were tightly encapsulated by flexible CCG sheets, thus allowing the close contact of CCG with soil and guaranteeing a sufficient dispersion. For comparison, we also characterized the blend of soil with MWCNTs by TEM. As shown in Fig. 2F, the tube-like structure of MWCNTs appeared more rigid than nanosheet of graphene, and the inner surfaces of MWCNTs were not available for sorption. Therefore, the degree of contact for MWCNTs with soil should be lower than that for CCG.

From the SEM and TEM measurements, it could be perceived that the nanosheet morphology of CCG made it suitable for use as sorbent and disperser. Furthermore, the mechanism of GA-MSPD should be somewhat different from that of normal MSPD. Since CCG was a relatively "soft" material, it provided little mechanical shearing to disrupt the coarse architecture of the sample; instead, this process should mainly be accomplished by the inter-particle shearing of the sample. However, CCG could provide a further dimension to the extraction: the lipophilic polyaromatic plane of CCG helped in dispersing different components of the sample matrix, which improved the extraction capability from some matrices.

\subsection{Quantification methods}

Before GA-MSPD experiment, the quantification methods for the target analytes were carefully selected. PBDEs and MeO-PBDEs were usually considered less polar than OH-PBDEs (as implied by $\log K_{\text {ow }}$ values in Table 1 ). GC-ECD was employed as a quantitative tool for PBDEs and MeO-PBDEs due to its higher sensitivity toward these brominated compounds than GC-MS [27]. A typical chromatogram of GC-ECD is given in Fig. S3. OH-PBDEs were usually determined by GC-MS after derivatization by diazomethane to their methoxylated analogs [28]. However, the derivatization step was time- and labor-consuming, and also involved carcinogenic reagent and might give rise to more errors in the quantification of the analytes. Thus, a LC-ESI-MS/MS method was developed herein for the quantification of $10 \mathrm{OH}$-PBDEs. Fig. S4 shows a typical chromatogram for OH-PBDEs. The combination of GC-ECD and LC-ESI-MS/MS ensured high sensitivity for all the tested compounds without derivatization.

\subsection{Optimization of GA-MSPD procedure}

The optimization of GA-MSPD procedure was conducted with a blank soil spiked with $2 \mathrm{ng} \mathrm{g}^{-1}$ PBDEs, MeO-PBDEs and $\mathrm{OH}-$ PBDEs (see Section 2.4 for detailed spiking procedure). The TOC of the soil sample was measured to be $0.89 \%$. Generally, two factors were taken into account: (1) extraction efficiency, which was indicated by the spike recoveries of the analytes; (2) co-eluted matrix components, which were reflected by interference peaks in the chromatogram. The type of eluent solvent was investigated firstly. The MSPD columns were eluted with $1 \mathrm{~mL}$ of six different eluents (hexane, DCM, acetone, acetonitrile (ACN), hexane/DCM 1:1 and hexane/acetone $1: 1$ ), and the recoveries were calculated by comparing the determined concentrations with the spiking concentration. Fig. 3A shows the mean recoveries of PBDEs, $\mathrm{MeO}-\mathrm{PBDEs}$ and $\mathrm{OH}-\mathrm{PBDEs}$. High recoveries of PBDEs and MeOPBDEs were obtained for all the tested eluents except hexane. However, DCM caused elevated baseline and ACN increased interference peaks in GC-ECD chromatograms. Thus, hexane/DCM, hexane/acetone and acetone were suitable for eluting PBDEs and MeO-PBDEs. Furthermore, because polar Florisil was used as cosorbent in the MSPD column and CCG still had many polar groups such as hydroxyl and carboxyl groups in its plane [5,9], OH-PBDEs yielded low recoveries with non-polar eluents due to the strong polar-polar interaction between $\mathrm{OH}-\mathrm{PBDEs}$ and the sorbents. Especially when hexane and hexane/DCM were used as eluents, scarcely any $\mathrm{OH}-\mathrm{PBDEs}$ were detected in the eluates. The most effective eluent for OH-PBDEs was acetone.

From the above results, we designed a two-step elution strategy to completely elute the analytes: firstly, PBDEs and MeOPBDEs were eluted with hexane/DCM; afterwards, OH-PBDEs that remained in the column were eluted with acetone. This strategy also matched the subsequent quantification methods. As shown in Fig. 1, the eluate of hexane/DCM containing PBDEs and MeO-PBDEs was subjected to GC-ECD analysis, while the eluate of acetone containing OH-PBDEs was analyzed by LC-ESI-MS/MS. This two-step elution strategy was used in all the following experiments.

We then studied the effect of CCG amount on the extraction efficiency. The results are shown in Fig. 3B. The CCG amount had no significant effect on the recoveries of PBDEs. However, the CCG 

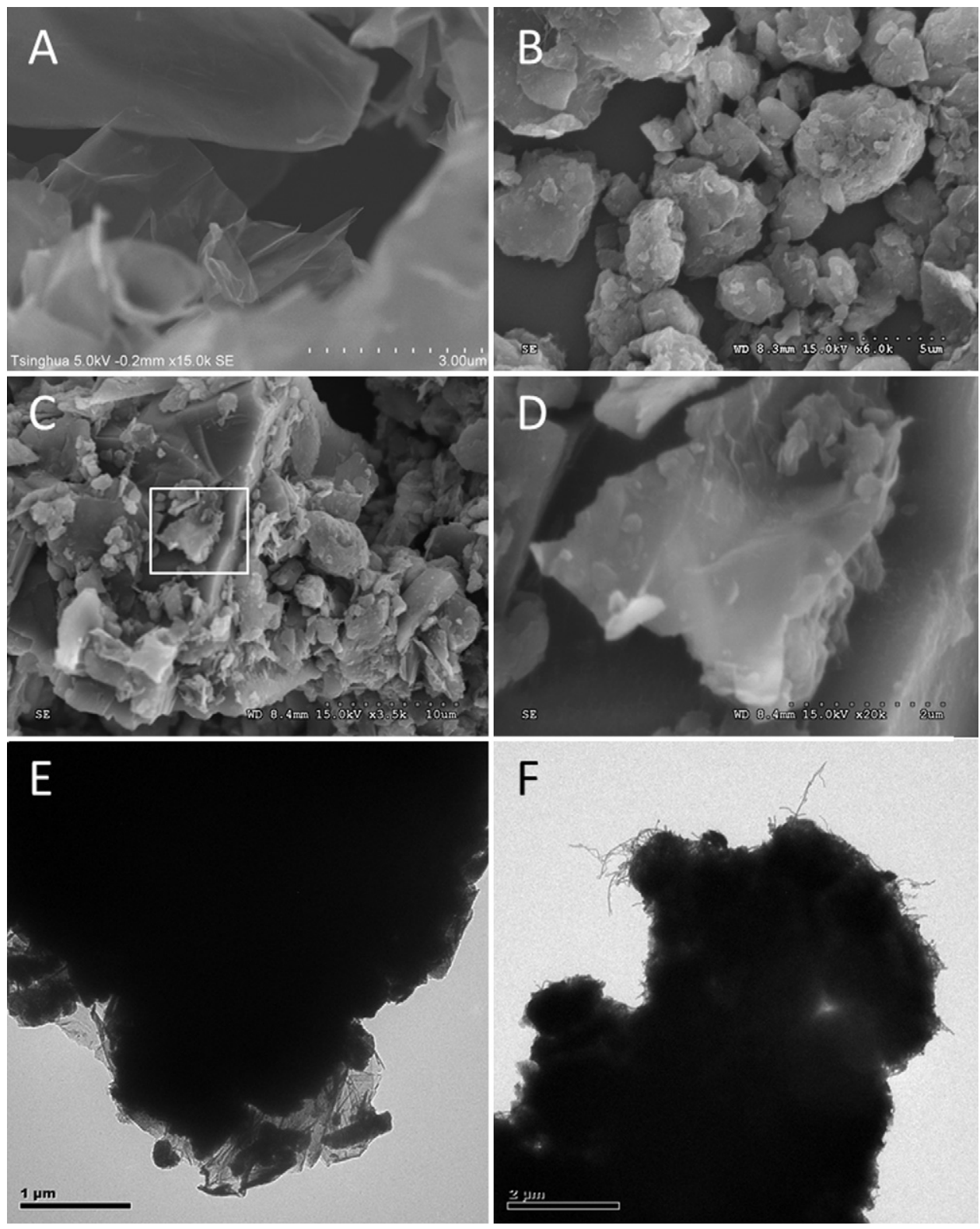

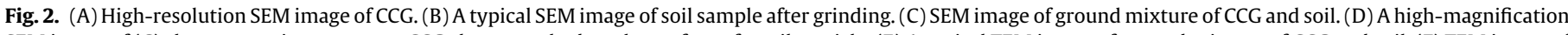

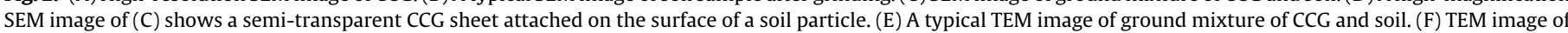
ground mixture of MWCNTs and soil.

amount played an important role in extracting OH-PBDEs. With increasing amount of CCG from 0 to $40 \mathrm{mg}$, the mean recoveries of OH-PBDEs markedly increased from $56.4 \%$ to $98.4 \%$. The possible reason was that the polar moieties of CCG could facilitate the dispersion of polar components of samples and extraction of the polar compounds. Furthermore, more interference peaks were observed with 0 or $5 \mathrm{mg}$ of CCG, revealing that another important role of CCG in GA-MSPD was to retain co-eluting matrix components. However, excessive amounts of CCG, e.g., $40 \mathrm{mg}$, would increase baseline noise probably because trace amount of CCG had been also eluted. Therefore, after carefully considering the recoveries and co-eluted matrix components, $10 \mathrm{mg}$ of CCG was used. Notably, in normal MSPD processes, about $0.5 \mathrm{~g}$ of sample was required and the ratio of sorbent to sample ranged from 1:1 to 4:1 [24]. However, in GAMSPD, the ratio of CCG to sample was 1:10, which was much lower than those in normal MSPD applications, mainly due to the high surface area and unique morphology of CCG.
Grinding time was optimized in the range 1-20 min. In fact, we found that grinding for $1 \mathrm{~min}$ readily resulted in an apparent homogeneous blend. As shown in Fig. 3C, comparable recoveries were achieved with prolonged grinding time from 1 to $20 \mathrm{~min}$. However, too rapid grinding would increase interference peaks due to insufficient retention of co-eluting matrix components. Thus, 5 min was adopted as an appropriate grinding time.

To further improve the extraction efficiency without increasing the consumption of solvent, the cycle number of elution was optimized [26]. The eluate was collected and reintroduced to the top of the column, and this process was repeated for 1,2 and 3 cycles. As shown in Fig. 3D, increasing the cycle number of elution was found to exert no effect on the extraction efficiency of PBDEs and MeOPBDEs. While for OH-PBDEs, increasing the elution cycle caused an adverse effect to the recoveries probably due to re-sorption of the analytes to the packing materials. Thus, a single elution step was conducted in further experiments of this work. 

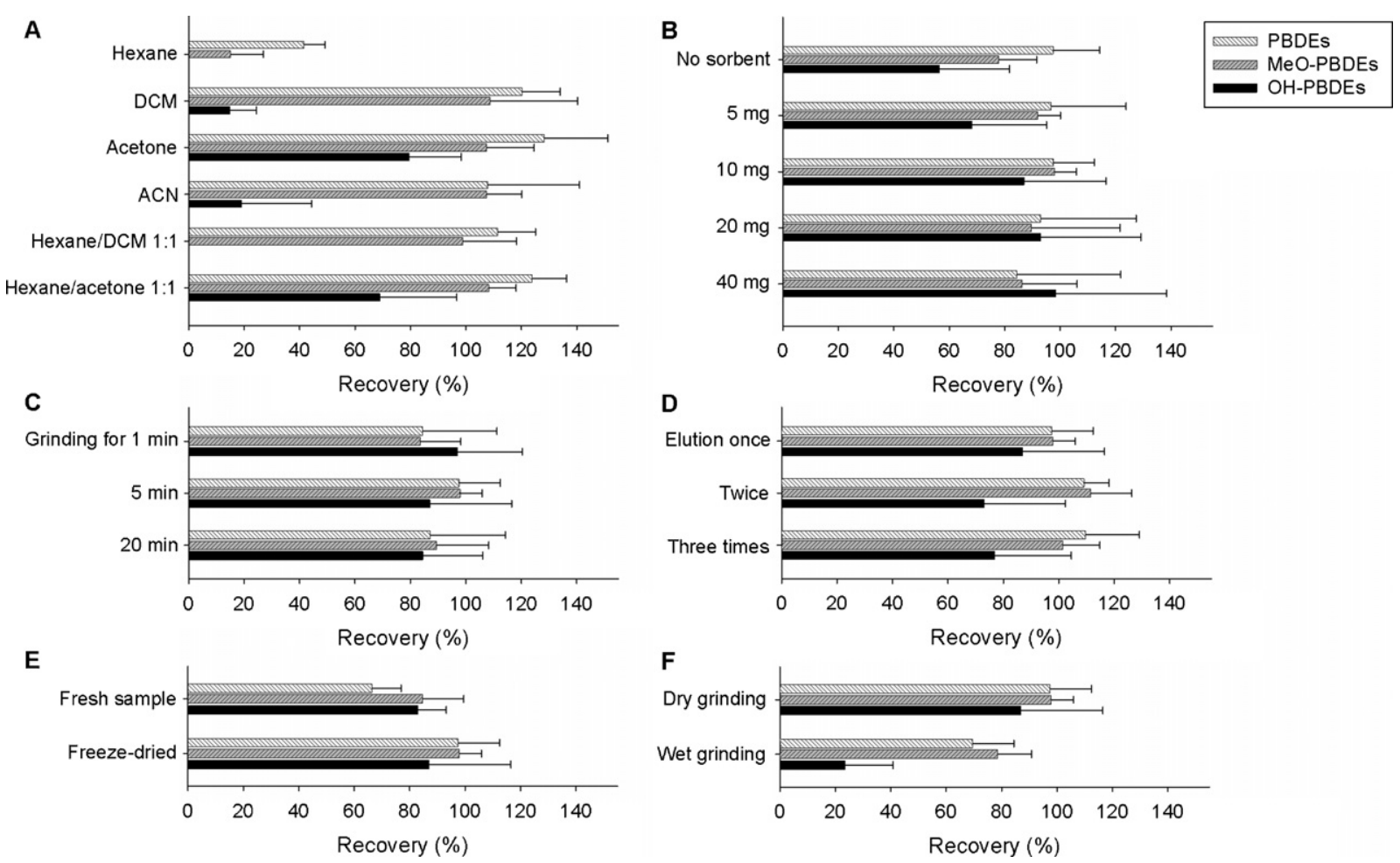

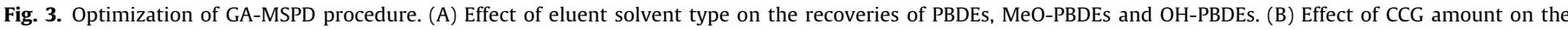

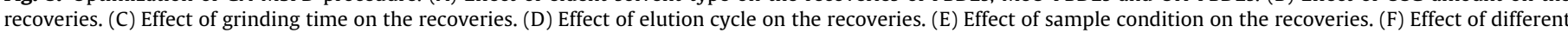

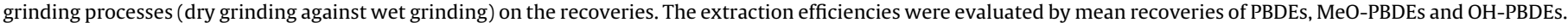
The error bars represent the standard deviations of recoveries among 5 PBDEs or 10 MeO-PBDEs or 10 OH-PBDEs.

Fig. 3E shows the extraction efficiencies with fresh and freezedried samples. Freeze-dried sample was more facile to handle and more easy to grind to homogeneous material with CCG. It also resulted in higher recoveries than fresh sample. Thus, freeze-dried samples were used in this work. However, it was worth pointing out that the recoveries obtained with fresh sample were also acceptable for quantitative analysis. Thus, this method still showed flexibility in analysis of samples of different conditions.

The wet grinding method was also tested by gradually adding $1 \mathrm{~mL}$ of hexane/acetone to the blend during grinding. Before elution, the sample was dried by air. As shown in Fig. 3F, wet grinding yielded lower recoveries than dry grinding, especially for $\mathrm{OH}-\mathrm{PBDEs}$. Furthermore, wet grinding increased the noise peaks observed in LC-MS/MS. Thus, dry grinding was chosen in this work. We have also found that increasing the dimension of column from 1 to $6 \mathrm{~mL}$ decreased the recoveries of OH-PBDEs (data not shown). Overall, under the optimized conditions, the mean recoveries of PBDEs, MeO-PBDEs and OH-PBDEs were 97.5, 97.9 and 87.0\%, respectively. In addition, we have found that this method was also effective for higher brominated PBDEs; e.g., in extraction of BDE$99,100,153,154$ and 183 from soil samples, the recoveries ranged from 77.6 to $114.7 \%$.

\subsection{Comparison studies}

The performance of GA-MSPD was compared with several other sorbent materials, including C18 silica, Florisil and MWCNTs, for extraction of PBDEs and their $\mathrm{MeO}-$ and $\mathrm{OH}-$ analogs from soil samples. At first, $100 \mathrm{mg}$ of spiked soil sample was blended with $10 \mathrm{mg}$ of sorbent in all cases. Preliminary studies showed that the two-step elution strategy was also applicable for the other sorbents studied. The results are shown in Fig. 4A. For PBDEs, the recoveries obtained with all the sorbents were satisfactory. However, for OH-PBDEs, the other sorbents yielded much poorer recoveries than CCG. Notably, the mean recovery of OH-PBDEs by MWCNTs was only $10.0 \%$. Note that MWCNTs had a similar polyaromatic structure to CCG but without polar groups, the low recoveries with MWCNTs also indicated that polar moieties of sorbent were important for extraction of polar compounds. Increasing the sorbent
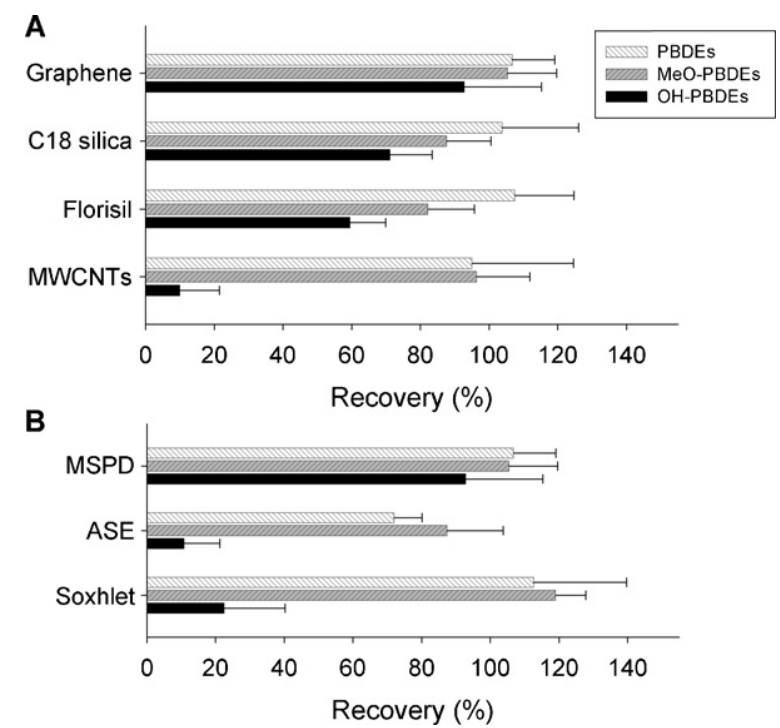

Fig. 4. (A) Comparison of CCG with other sorbents (C18, Florisil and MWCNTs) for extraction of PBDEs, MeO-PBDEs and OH-PBDEs from soils. The sorbent amount was $20 \mathrm{mg}$ in all cases. (B) Comparison of GA-MSPD with other extraction techniques (ASE and Soxhlet extraction). For PBDEs and MeO-PBDEs in ASE and Soxhlet extraction, the extracts were purified by using sulfuric acid before GC-ECD analysis; while for OH-PBDEs, the extracts were directly analyzed by LC-ESI-MS/MS without any other cleanup step. 
Table 1

Analytical figures of merit in analysis of soil samples.

\begin{tabular}{|c|c|c|c|c|c|c|c|c|}
\hline \multirow[t]{2}{*}{ Compound } & \multirow[t]{2}{*}{$\log K_{\mathrm{ow}}$} & \multirow[t]{2}{*}{$\mathrm{p} K_{\mathrm{a}}$} & \multirow[t]{2}{*}{ Detection } & \multirow[t]{2}{*}{ Linear range $\left(\mathrm{ng} \mathrm{g}^{-1}\right)$} & \multirow[t]{2}{*}{$r^{2 c}$} & \multirow[t]{2}{*}{$\operatorname{MDL}\left(\mathrm{pg} \mathrm{g}^{-1}\right)^{\mathrm{d}}$} & \multicolumn{2}{|l|}{ RSD (\%) } \\
\hline & & & & & & & Run-to-run ${ }^{\mathrm{e}}$ & Day-to-dayf \\
\hline BDE-17 & $5.74 \pm 0.22^{\mathrm{a}}$ & - & GC-ECD & $0.1-50.0$ & 0.9920 & 28.7 & 5.7 & 12.1 \\
\hline BDE-28 & $5.94 \pm 0.15^{a}$ & - & GC-ECD & $0.1-50.0$ & 0.9827 & 10.0 & 6.7 & 6.3 \\
\hline BDE-47 & $6.81 \pm 0.08^{a}$ & - & GC-ECD & $0.1-50.0$ & 0.9938 & 18.2 & 9.6 & 12.8 \\
\hline BDE-66 & - & - & GC-ECD & $0.1-50.0$ & 0.9916 & 8.1 & 5.9 & 15.7 \\
\hline BDE-85 & $7.37 \pm 0.12^{\mathrm{a}}$ & - & GC-ECD & $0.1-50.0$ & 0.9938 & 5.9 & 8.3 & 14.3 \\
\hline 3'-MeO-BDE-28 & - & - & GC-ECD & $0.1-50.0$ & 0.9908 & 14.7 & 7.4 & 8.9 \\
\hline 4-MeO-BDE-42 & - & - & GC-ECD & $0.1-50.0$ & 0.9964 & 32.8 & 15.4 & 7.2 \\
\hline 3-MeO-BDE-47 & - & - & GC-ECD & $0.1-50.0$ & 0.9962 & 20.6 & 12.3 & 10.0 \\
\hline 5-MeO-BDE-47 & - & - & GC-ECD & $0.1-50.0$ & 0.9958 & 23.6 & 11.2 & 13.6 \\
\hline 6-MeO-BDE-47 & $6.44 \pm 0.01^{\mathrm{b}}$ & - & GC-ECD & $0.1-50.0$ & 0.9960 & 20.2 & 9.9 & 12.7 \\
\hline $4^{\prime}-\mathrm{MeO}-\mathrm{BDE}-49$ & - & - & GC-ECD & $0.1-50.0$ & 0.9968 & 46.6 & 10.1 & 17.9 \\
\hline $2^{\prime}-\mathrm{MeO}-\mathrm{BDE}-68$ & $6.16 \pm 0.02^{\mathrm{b}}$ & - & GC-ECD & $0.1-50.0$ & 0.9920 & 14.3 & 10.9 & 8.3 \\
\hline 6-MeO-BDE-85 & $6.26 \pm 0.01^{\mathrm{b}}$ & - & GC-ECD & $0.1-50.0$ & 0.9906 & 15.1 & 9.1 & 19.8 \\
\hline 5'-MeO-BDE-99 & - & - & GC-ECD & $0.1-50.0$ & 0.9956 & 20.2 & 4.9 & 12.4 \\
\hline $6^{\prime}-\mathrm{MeO}-\mathrm{BDE}-99$ & - & - & GC-ECD & $0.1-50.0$ & 0.9857 & 18.3 & 5.5 & 18.7 \\
\hline $3^{\prime}-\mathrm{OH}-\mathrm{BDE}-28$ & - & - & LC-MS/MS & $0.5-50.0$ & 0.9916 & $2.1 \times 10^{2}$ & 9.7 & 18.6 \\
\hline 4-OH-BDE-42 & - & - & LC-MS/MS & $0.2-50.0$ & 0.9750 & 49.8 & 7.0 & 12.0 \\
\hline 3-OH-BDE-47 & - & - & LC-MS/MS & $0.2-50.0$ & 0.9938 & 64.6 & 9.5 & 3.7 \\
\hline 5-OH-BDE-47 & - & - & LC-MS/MS & $0.1-50.0$ & 0.9870 & 5.3 & 6.6 & 15.4 \\
\hline $6-\mathrm{OH}-\mathrm{BDE}-47$ & $5.82 \pm 0.03^{b}$ & $6.8 \pm 0.2^{\mathrm{b}}$ & LC-MS/MS & $0.2-50.0$ & 0.9964 & 44.4 & 5.3 & 7.8 \\
\hline $4^{\prime}-\mathrm{OH}-\mathrm{BDE}-49$ & - & - & LC-MS/MS & $0.2-50.0$ & 0.9842 & 56.5 & 8.2 & 5.6 \\
\hline $2^{\prime}-\mathrm{OH}-\mathrm{BDE}-68$ & $5.36 \pm 0.04^{\mathrm{b}}$ & $6.6 \pm 0.2^{b}$ & LC-MS/MS & $0.1-50.0$ & 0.9886 & 33.9 & 5.1 & 14.1 \\
\hline 6-OH-BDE-85 & $5.83 \pm 0.02^{\mathrm{b}}$ & $6.2 \pm 0.2^{\mathrm{b}}$ & LC-MS/MS & $0.2-50.0$ & 0.9944 & 59.8 & 4.9 & 9.6 \\
\hline 5'-OH-BDE-99 & - & - & LC-MS/MS & $0.2-50.0$ & 0.9948 & 91.2 & 7.3 & 12.0 \\
\hline 6'-OH-BDE-99 & - & - & LC-MS/MS & $0.1-50.0$ & 0.9829 & 35.3 & 2.7 & 9.9 \\
\hline
\end{tabular}

a The data were from Ref. [38].

b The data were from Ref. [39].

c $r=$ correlative coefficient.

d MDLs were calculated following the US EPA recommended procedure, in $\operatorname{pg~g}^{-1}$ dry weight.

e The run-to-run RSDs were calculated based on five successive extractions.

f The day-to-day RSDs were obtained over 4 days.

amount of C18, Florisil and MWCNTs to $100 \mathrm{mg}$ caused no positive effect on the recoveries (Fig. S5). This suggested that the co-sorbent Florisil might also play an important role in compound fractionation. Conclusively, the comparison with other sorbents has verified that CCG is a highly efficient sorbent in handling solid samples.

We also compared the GA-MSPD with other extraction techniques, including accelerated solvent extraction (ASE) and Soxhlet extraction. The optimized conditions of ASE and Soxhlet were presented in Supporting Information. Unlike GA-MSPD, ASE and Soxhlet are much more harsh extraction techniques in that large amounts of matrix components are simultaneously extracted. Thus, their extracts were subjected to further purification (i.e., using sulfuric acid [29-31]) before GC-ECD analysis. Fig. 4B shows the recoveries obtained with other extraction techniques. For PBDEs and MeO-PBDEs, GA-MSPD and Soxhlet offered comparable recoveries, and ASE yielded somewhat lower recoveries. While the mean recoveries of OH-PBDEs obtained in ASE and Soxhlet were only 10.8 and $22.5 \%$, respectively, probably because phenolic compounds were not efficiently extracted into neutral organic solvents when they were in an ionized form [32]. This situation could be improved by adding acid to the extraction solvent [32], but this would add extra cleanup steps to the method. By contrast, GA-MSPD yielded high recoveries for $\mathrm{OH}-\mathrm{PBDEs}$ due to the strong sorption and dispersing ability of CCG.

From the above results, the advantages of GA-MSPD mainly lie in: (1) Higher recoveries for analytes with a wide range of polarity. (2) Short extraction time. The extraction process is shortened from ca. $24 \mathrm{~h}$ for Soxhlet and ca. $1 \mathrm{~h}$ for ASE to ca. $15 \mathrm{~min}$ for GA-MSPD. (3) Minimized consumption of sorbent and solvent. In this study, only $10 \mathrm{mg}$ of CCG and $2 \mathrm{~mL}$ of organic solvents are used in each extraction. The sample amount $(100 \mathrm{mg})$ was also reduced compared with ASE and Soxhlet as well as normal MSPD methods.

\subsection{Extraction performance}

The analytical figures of merit of the GA-MSPD method with soil as model sample matrix were presented in the Table 1 . To eliminate any possible matrix effect, the seven-point calibration curves were constructed in sample matrices in the range of $0.1-50.0$ ng g $^{-1}$ dry weight. Good linearities were obtained for all the tested compounds $\left(R^{2} 0.9750-0.9968\right)$. The method detection limits (MDLs) were determined according to the standards procedure recommended by US EPA [33]. The detailed process was given in the Supporting Information. The obtained MDLs of PBDEs, MeO-PBDEs and OH-PBDEs were in the range of 5.9-28.7, $14.3-46.6$, and $5.3-212.6$ pg g $^{-1}$ dry weight, respectively. The sensitivity of PBDEs was at the same level as that obtained with pressurized liquid extraction coupled to GC-MS [34] and hollow fiber-liquid phase microextraction with GC-ICP MS [35], and it was also slightly higher than that obtained with SPME with GC-MS [36]. For OH-PBDEs, the sensitivity was better than or comparable to that obtained with ultrasonic-assisted extraction followed by LC-negative ion-spray ionization-MS/MS [29]. The precision of the method was estimated using soil samples spiked with standards at $1 \mathrm{ng} \mathrm{g}^{-1}$. As listed in Table 1, the run-to-run and day-to-day RSDs were in the range of $2.7-15.4 \%$ and $3.7-19.8 \%$, respectively.

\subsection{Applications to different environmental samples}

The proposed method was validated with different environmental samples, including soil, tree bark, and fish. These samples were all collected from an E-waste dismantling area where heavy contamination of PBDEs has been found [37]. Typical chromatograms from analysis of soil samples were given in Fig. S6. For tree bark and fish samples, due to their rather complex matrices, the extracts were purified using sulfuric acid before GC and LC analysis [29-31]. 
Table 2

Concentrations $\left(\mathrm{ng} \mathrm{g}^{-1}\right)$ found in and spike recoveries (\%) from real environmental samples $(n=3)$.

\begin{tabular}{|c|c|c|c|c|c|c|c|c|c|c|c|c|}
\hline \multirow[t]{2}{*}{ Compound } & \multicolumn{4}{|l|}{ Soil } & \multicolumn{4}{|c|}{ Tree bark } & \multicolumn{4}{|l|}{ Fish } \\
\hline & $\begin{array}{l}\text { Found } \\
\left(\mathrm{ng} \mathrm{g}^{-1}\right)\end{array}$ & $\begin{array}{l}\text { Spike } \\
0.25 \text { ng g }^{-1}\end{array}$ & $\begin{array}{l}\text { Spike } \\
1 \mathrm{ng} \mathrm{g}^{-1}\end{array}$ & $\begin{array}{l}\text { Spike } \\
5 \mathrm{ng} \mathrm{g}^{-1}\end{array}$ & $\begin{array}{l}\text { Found } \\
\left(\mathrm{ng} \mathrm{g}^{-1}\right)\end{array}$ & $\begin{array}{l}\text { Spike } \\
1 \text { ng g }^{-1}\end{array}$ & $\begin{array}{l}\text { Spike } \\
5 \mathrm{ng} \mathrm{g}^{-1}\end{array}$ & $\begin{array}{l}\text { Spike } \\
10 \text { ng g }^{-1}\end{array}$ & $\begin{array}{l}\text { Found } \\
\left(\mathrm{ng} \mathrm{g}^{-1}\right)\end{array}$ & $\begin{array}{l}\text { Spike } \\
1 \text { ng g }^{-1}\end{array}$ & $\begin{array}{l}\text { Spike } \\
5 \mathrm{ng} \mathrm{g}^{-1}\end{array}$ & $\begin{array}{l}\text { Spike } \\
10 \mathrm{ngg}^{-1}\end{array}$ \\
\hline BDE-17 & $\mathrm{nd}^{\mathrm{a}}$ & 102.4 & 103.5 & 69.4 & 6.0 & 109.0 & 108.5 & 87.4 & nd & 84.0 & 87.6 & 58.2 \\
\hline BDE-28 & 0.3 & 77.0 & 82.4 & 69.8 & 5.0 & 89.0 & 94.2 & 77.3 & 4.7 & 86.0 & 91.6 & 55.9 \\
\hline BDE-47 & 0.2 & 101.6 & 94.1 & 89.7 & 13.5 & $112.4^{\mathrm{c}}$ & 88.8 & 62.2 & nd & 114.1 & 93.7 & 61.0 \\
\hline BDE-66 & nd & 89.7 & 104.6 & 99.6 & nd & I & I & I & nd & I & 69.0 & 48.3 \\
\hline BDE-85 & nd & 116.0 & 101.2 & 92.5 & 1.9 & 75.0 & 78.0 & 70.4 & nd & 93.5 & 88.7 & 60.7 \\
\hline 3'-MeO-BDE-28 & nd & $\mathrm{I}^{\mathrm{b}}$ & I & 96.5 & nd & 88.1 & 78.3 & 90.8 & nd & 88.2 & 72.7 & 47.1 \\
\hline 4-MeO-BDE-42 & nd & 118.8 & 113.6 & 93.1 & nd & 77.1 & 82.8 & 107.4 & nd & 64.6 & 84.3 & 58.1 \\
\hline 3-MeO-BDE-47 & nd & 101.9 & 109.5 & 98.1 & nd & I & I & I & nd & 74.2 & 82.4 & 52.3 \\
\hline 5-MeO-BDE-47 & nd & 108.9 & 112.7 & 99.3 & nd & I & I & I & nd & 75.4 & 87.7 & 55.9 \\
\hline 6-MeO-BDE-47 & nd & 112.2 & 118.2 & 93.7 & nd & 78.7 & 87.1 & 66.3 & nd & I & 109.9 & 63.6 \\
\hline 4'-MeO-BDE-49 & nd & 123.5 & 112.6 & 97.1 & nd & 60.1 & 69.8 & 60.0 & nd & 61.9 & 69.8 & 50.3 \\
\hline 2'-MeO-BDE-68 & nd & 91.4 & 91.1 & 88.9 & nd & 85.6 & 91.0 & 67.1 & nd & I & 80.3 & 50.9 \\
\hline 6-MeO-BDE-85 & nd & 125.9 & 108.8 & 103.4 & nd & 81.8 & 74.8 & 99.8 & nd & 91.3 & 78.5 & 57.9 \\
\hline 5'-MeO-BDE-99 & nd & 106.9 & 95.4 & 98.0 & nd & 84.4 & 72.8 & 78.4 & nd & 79.9 & 80.0 & 54.3 \\
\hline 6'-MeO-BDE-99 & nd & 102.9 & 106.5 & 96.1 & nd & 79.0 & 86.7 & 71.3 & nd & 109.3 & 89.1 & 56.7 \\
\hline 3'-OH-BDE-28 & nd & 88.4 & 118.3 & 95.7 & nd & 72.8 & 51.5 & 53.6 & nd & 33.7 & 35.0 & 23.8 \\
\hline 4-OH-BDE-42 & nd & 104.3 & 106.0 & 93.5 & nd & 77.8 & 74.1 & 81.5 & nd & 29.1 & 29.9 & 28.6 \\
\hline 3-OH-BDE-47 & nd & 115.4 & 110.1 & 92.3 & nd & 67.9 & 72.3 & 86.8 & nd & 66.4 & 51.5 & 48.9 \\
\hline 5-OH-BDE-47 & nd & 70.5 & 97.6 & 105.7 & nd & 59.6 & 68.5 & 84.6 & nd & 57.6 & 31.1 & 28.7 \\
\hline 6-OH-BDE-47 & nd & 112.5 & 109.2 & 90.1 & nd & 94.3 & 88.9 & 93.3 & nd & 76.8 & 49.3 & 46.5 \\
\hline $4^{\prime}-\mathrm{OH}-\mathrm{BDE}-49$ & nd & 83.4 & 109.4 & 91.7 & nd & 60.7 & 89.1 & 82.9 & nd & 30.4 & 31.6 & 27.9 \\
\hline $2^{\prime}-\mathrm{OH}-\mathrm{BDE}-68$ & nd & 88.9 & 113.3 & 97.5 & nd & 90.9 & 113.6 & 94.1 & nd & 59.8 & 71.9 & 67.7 \\
\hline 6-OH-BDE-85 & nd & 86.0 & 97.8 & 103.5 & nd & 76.4 & 87.0 & 100.4 & nd & 81.2 & 59.3 & 68.6 \\
\hline 5'-OH-BDE-99 & nd & 96.8 & 100.3 & 105.8 & nd & 73.4 & 93.7 & 84.6 & nd & 69.6 & 46.6 & 34.1 \\
\hline 6'-OH-BDE-99 & nd & 116.0 & 112.7 & 102.9 & nd & 82.7 & 86.4 & 90.8 & nd & 87.1 & 77.4 & 85.3 \\
\hline
\end{tabular}

a nd, not detected.

b I, quantitation was interfered by unknown peaks.

c The numbers in italics carry higher uncertainties.

The PBDEs, MeO-PBDEs and OH-PBDEs in the samples were determined first. Then, three concentration levels of standards were spiked to the samples and the spike recoveries were examined. The measurements were repeated for three times. The results are listed in Table 2. It can be seen that PBDEs could be detected in these samples, with some congeners at relatively high concentrations (e.g., BDE-47 in tree bark). No MeO-PBDEs or OH-PBDEs were found in these samples. For soil samples, the spike recoveries of the target analytes ranged from 69.4 to $125.9 \%$. Tree bark appeared to be a more complex matrix than soil, and several analytes (BDE-66, 3-MeO-BDE-47 and 5-MeO-BDE-47) were co-eluted with unknown peaks. However, satisfactory recoveries of the other analytes were obtained (51.5-112.4\%). For fish samples, high spike recoveries (61.9-114.1\%) were achieved for PBDEs and MeO-PBDEs at the spike levels of 1 and $5 \mathrm{ngg}^{-1}$. While at the spike level of $10 \mathrm{ng} \mathrm{g}^{-1}$ and for OH-PBDEs, somewhat lower recoveries were obtained (23.8-87.1\%). The possible reason might be that fish was a relatively soft sample matrix, which might result in incomplete homogenization with CCG. Another possible explanation was that fish had high lipid content, and thus more depletion of analytes might occur during the cleanup step. Despite that, the extraction efficiency was generally acceptable for quantitative analysis. Therefore, GA-MSPD was suitable for the extraction of PBDEs and their $\mathrm{MeO}-$ and $\mathrm{OH}$ - analogs in different environmental matrices, especially in relatively hard and abrasive samples.

\section{Conclusions}

CCG has been shown for the first time to be a useful sorbent and dispersing material in handling solid samples. A GA-MSPD method has been developed for the simultaneous extraction of PBDEs and their $\mathrm{MeO}-$ and $\mathrm{OH}-$ analogs from environmental matrices. Compared with other sorbents and extraction techniques, GA-MSPD possessed advantages such as high recoveries, reduced use of sorbent, low consumption of sample and organic solvent, simple extraction procedure, and short extraction time. By coupling with GC-ECD and LC-ESI-MS/MS, satisfactory sensitivity and reproducibility were achieved for the target compounds. The method was finally successfully applied to the analysis of PBDEs, MeOPBDEs and OH-PBDEs in real environmental samples, including soil, tree bark and fish. Therefore, it is believed that GA-MSPD is a promising tool for pretreatment of solid or semisolid samples.

\section{Acknowledgements}

This work was jointly supported by the National Basic Research Program of China (No. 2009CB421605), the National Natural Science Foundation of China (No. 21107120, 20890111, 20921063 and 20931160427), and the China Postdoctoral Science Foundation (No. 20100470024).

\section{Appendix A. Supplementary data}

Supplementary data associated with this article can be found, in the online version, at doi:10.1016/j.aca.2011.09.017.

\section{References}

[1] K.S. Novoselov, A.K. Geim, S.V. Morozov, D. Jiang, Y. Zhang, S.V. Dubonos, I.V. Grigorieva, A.A. Firsov, Science 306 (2004) 666-669.

[2] Y. Zhu, S. Murali, W. Cai, X. Li, J.W. Suk, J.R. Potts, R.S. Ruoff, Adv. Mater. 22 (2010) 3906-3924.

[3] A.K. Geim, Science 324 (2009) 1530-1534.

[4] M.D. Stoller, S.J. Park, Y.W. Zhu, J.H. An, R.S. Ruoff, Nano Lett. 8 (2008) 3498-3502.

[5] S. Stankovich, D.A. Dikin, R.D. Piner, K.A. Kohlhaas, A. Kleinhammes, Y. Jia, Y. Wu, S.T. Nguyen, R.S. Ruoff, Carbon 45 (2007) 1558-1565.

[6] S. Park, R.S. Ruoff, Nat. Nanotechnol. 4 (2009) 217-224.

[7] V. Chandra, J. Park, Y. Chun, J.W. Lee, I.C. Hwang, K.S. Kim, ACS Nano 4 (2010) 3979-3986.

[8] S.T. Yang, Y.L. Chang, H.F. Wang, G.B. Liu, S. Chen, Y.W. Wang, Y.F. Liu, A.N. Cao, J. Colloid Interface Sci. 351 (2010) 122-127.

[9] Q. Liu, J. Shi, L. Zeng, T. Wang, Y. Cai, G. Jiang, J. Chromatogr. A 1218 (2011) 197-204. 
[10] Q. Liu, J. Shi, J. Sun, T. Wang, L. Zeng, G. Jiang, Angew. Chem. Int. Ed. 50 (2011) 5913-5917.

[11] X.L. Dong, J.S. Cheng, J.H. Li, Y.S. Wang, Anal. Chem. 82 (2010) 6208-6214.

[12] L.A.L. Tang, J.Z. Wang, K.P. Loh, J. Am. Chem. Soc. 132 (2010) 10976-10977.

[13] J.M. Chen, J. Zou, J.B. Zeng, X.H. Song, J.J. Ji, Y.R. Wang, J. Ha, X. Chen, Anal. Chim. Acta 678 (2010) 44-49.

[14] Y.-B. Luo, J.-S. Cheng, Q. Ma, Y.-Q. Feng, J.-H. Li, Anal. Methods 3 (2011) 92-98.

[15] H. Zhang, H.K. Lee, J. Chromatogr. A 1218 (2011) 4509-4516.

[16] I. Watanabe, S. Sakai, Environ. Int. 29 (2003) 665-682

[17] R.A. Hites, Environ. Sci. Technol. 38 (2004) 945-956.

[18] A. Malmvarn, G. Marsh, L. Kautsky, M. Athanasiadou, A. Bergman, L. Asplund, Environ. Sci. Technol. 39 (2005) 2990-2997.

[19] S. Lacorte, M.G. Ikonomou, Chemosphere 74 (2009) 412-420.

[20] M. Athanasiadou, S.N. Cuadra, G. Marsh, A. Bergman, K. Jakobsson, Environ. Health Perspect. 116 (2008) 400-408.

[21] I.A.T.M. Meerts, R.J. Letcher, S. Hoving, G. Marsh, A. Bergman, J.G. Lemmen, B. van der Burg, A. Brouwer, Environ. Health Perspect. 109 (2001) 399-407.

[22] R.F. Song, T.L. Duarte, G.M. Almeida, P.B. Farmer, M.S. Cooke, W.B. Zhang, G.Y. Sheng, J.M. Fu, G.D.D. Jones, Toxicol. Lett. 185 (2009) 23-31.

[23] S.A. Barker, J. Chromatogr. A 885 (2000) 115-127.

[24] A.L. Capriotti, C. Cavaliere, P. Giansanti, R. Gubbiotti, R. Samperi, A. Lagana, J. Chromatogr. A 1217 (2010) 2521-2532.

[25] W.S. Hummers, R.E. Offeman, J. Am. Chem. Soc. 80 (1958) 1339.

[26] R.M.A.P.S. Dassanayake, H. Wei, R.C. Chen, A. Li, Anal. Chem. 81 (2009) 9795-9801.
[27] A. Martinez, M. Ramil, R. Montes, D. Hernanz, E. Rubi, I. Rodriguez, R.C. Torrijos, J. Chromatogr. A 1072 (2005) 83-91.

[28] G. Marsh, M. Athanasiadou, A. Bergman, L. Asplund, Environ. Sci. Technol. 38 (2004) 10-18.

[29] S. Mas, O. Jáuregui, F. Rubio, A.D. Juan, R. Tauler, S. Lacorte, J. Mass Spectrom. 42 (2007) 890-899.

[30] L.Y. Zhu, B.L. Ma, M.W. Liang, Rapid Commun. Mass Spectrom. 22 (2008) 394-400.

[31] P.S. Haglund, D.R. Zook, H.-R. Buser, J. Hu, Environ. Sci. Technol. 31 (1997) 3281-3287.

[32] H. Chang, Y. Wan, J. Naile, X.W. Zhang, S. Wiseman, M. Hecker, M.H.W. Lam, J.P. Giesy, P.D. Jones, J. Chromatogr. A 1217 (2010) 506-513.

[33] Test Methods for Evaluating Solid Waste, Physical/Chemical Methods (SW846), Chapter 1, Quality Control, United States Environmental Protection Agency, http://www.epa.gov/epawaste/hazard/testmethods/sw846/online/ index.htm.

[34] Z.L. Zhang, M. Shanmugam, S.M. Rhind, Chromatographia 72 (2010) 535-543.

[35] Q. Xiao, B. Hu, J.K. Duan, M. He, W.Q. Zu, J. Am. Soc. Mass Spectrom. 18 (2007) 1740-1748

[36] J.J. Zhou, F.X. Yang, D.M. Cha, Z.R. Zeng, Y. Xu, Talanta 73 (2007) 870-877.

[37] J. Fu, Y. Wang, A. Zhang, Q. Zhang, Z. Zhao, T. Wang, G. Jiang, Chemosphere 82 (2011) 648-655.

[38] E. Braekevelt, S.A. Tittlemier, G.T. Tomy, Chemosphere 51 (2003) 563-567.

[39] K. Löfstrand, Trends and exposure of naturally produced brominated substances in Baltic biota, Thesis, Department of Materials and Environmental Chemistry, Stockholm University, Stockholm, 2011. 